\title{
The effects of catalyst introduction in carbon fiber coating process via CVD on tensile properties of carbon fiber reinforced polypropylene composite
}

\begin{abstract}
The tensile property of carbon nanotube coated carbon fibre was studied using the tensile test measurement. The surface of the carbon fibres were coated with carbon nanotubes (CNTs) through coating process using catalyst chemical vapour deposition (CVD) apparatus. In the present investigation, ferrocene has been used as the catalyst precursor to synthesize CNT on the surface of the carbon fibre. The way in which ferrocene has been introduced into CVD during coating process, however, gives significant different in CNTs morphology which resulting different performance in tensile properties of carbon fibre reinforced polypropylene (CFPP). The strong adhesion between the CNT and carbon fibre surface improved the tensile strength and tensile modulus of the CFPP laminates approximately to $36 \%$ and $100 \%$ respectively compare to the uncoated CFPP composites.
\end{abstract}

